\title{
Diferencias Morfométricas en el Hueso Palatino de la Oveja (Ovis aries) y la Cabra (Capra hircus)
}

\author{
Morphometric Palatine Differences Between Sheep (Ovis aries) and Goat (Capra hircus)
}

Pere M. Parés-Casanova ${ }^{1}$; Xénia Domènech ${ }^{1}$; Concepción Rojo² \& Fidel San Román ${ }^{3}$

PARÉS-CASANOVA, P. M.; DOMÈNECH, X.; ROJO, C. \& SAN ROMÁN, F. Diferencias morfométricas en el hueso palatino de la oveja (Ovis aries) y la cabra (Capra hircus). Int. J. Morphol., 39(3):797-801, 2021.

RESUMEN: Un hueso craneal muy poco estudiado en anatomía veterinaria comparada es el palatino. En nuestro estudio, realizamos la comparación de este hueso entre la oveja (Ovis aries L., 1758) y la cabra (Capra hircus L., 1758), a partir de una muestra de 36 y 17 cráneos respectivamente, y utilizando métodos de morfometría geométrica. Se eligieron un total de 32 puntos (4 hitos y 28 semi-hitos) para analizar la lámina horizontal del hueso palatino. Se reflejaron diferencias estadísticamente significativas tanto para el tamaño como para la forma. En Capra se denota una clara expansión lateral del hueso y rostral y una contracción central, con un acercamiento relativo de los forámenes palatinos, mientras que en Ovis los forámenes están más lateralmente alejados. A nuestro parecer, esta mayor anchura y longitud de la lámina horizontal en Capra se explicaría por una mayor capacidad de frotación del alimento en relación a Ovis.

PALABRAS CLAVE: Raza Blanca de Rasquera; Esplacnocráneo; Paladar duro; Raza Navarra; Viscerocráneo.

\section{INTRODUCCIÓN}

Probablemente el cráneo es una de las estructuras más estudiadas en los animales domésticos, tanto desde el punto de vista de pura descripción anatómica (Rodrigues et al., 2010; Merkies et al., 2020), comparativo (Burcu et al., 2013), functional (Evans \& McGreevy, 2006; Dalga, 2020), ontogenético (Geiger \& Haussman, 2016) y, especialmente, etnológico (Schoenebeck \& Ostrander, 2013; Goodarzi \& Hoseini, 2014; Parés-Casanova, 2015; Choudhary et al., 2020).

En este sentido, resulta importante determinar y analizar los componentes esqueléticos del cráneo para las diferentes especies domésticas. La cavidad de la cavidad oral tiene un techo óseo que es el paladar, y sus límites laterales los constituyen los márgenes maxilar y mandibular junto con las encías y dientes. La base ósea del paladar duro [Palatum osseum] la forman los procesos palatinos del hueso incisivo (rostralmente) y del hueso maxilar, así como el hueso palatino [Os palatinum] caudalmente.
El hueso palatino es un hueso par, situado entre el hueso maxilar, el hueso esfenoides y el hueso pterigoides (König \& Liebich, 2005). Cada hueso palatino se articula con el contralateral, el hueso maxilar, el esfenoides, el etmoides, la concha nasal ventral y el vómer. El hueso palatino está compuesto por una lámina horizontal [Lamina horizontalis] y una vertical [Lamina perpendicularis] (König \& Liebich). En rumiantes, la lámina horizontal es muy ancha, y contiene,junto con el proceso palatino del maxilar,el seno palatino [Sinus palatinus] (König \& Liebich). La lámina horizontal forma la parte caudal del paladar duro (Gioso \& Carvalho, 2005), y está sagitalmente marcada por la sutura palatina [Sutura palatina mediana] (König \& Liebich). Presenta en rumiantes el canal palatino mayor [Canalis palatinus major] a ambos lados, que desde los forámenes palatinos mayores [Foramen palatinum majus] conducen la arteria y el nervio palatinos mayores hacia el paladar (Sisson \& Grossman, 1985). También pueden estar presentes algunos forámenes palatinos menores, paso de arterias palatinas

\footnotetext{
${ }^{1}$ Departamento de Ciencia Animal, ETSEA, Universidad de Lleida. Av. Rovira Roure 191, 25198 Lleida, Cataluña, España.

${ }^{2}$ Sección Departamental de Anatomía y Embriología, Universidad Complutense Madrid. Avda. Puerta de Hierro S/N 28040, Madrid, España.

${ }^{3}$ Departamento de Medicina y Cirugía Animal. Facultad de Veterinaria, Universidad Complutense Madrid. Avda. Puerta de Hierro S/N 28040, Madrid, España.
} 
menores (Gioso \& Carvalho). El margen caudal de la lámina horizontal [Margo liber] no contacta con ningún hueso, y presenta medialmente una proyección cónica de dirección caudal que forma la espina nasal caudal(Sisson \& Grossman; König \& Liebich). El músculo tensor del velo del paladar se fija a lo largo de este margen mediante la fuerte aponeurosis del velo del paladar (Sisson \& Grossman).

Si bien el hueso palatino ha sido profundamente estudiado en medicina humana, en parte por sus implicaciones en problemas clínicos sobre todo ortodóncicos (Iseri et al., 1998; Gedrange et al., 2001; Lee et al., 2001; Laganà et al., 2019), en anatomía animal su estudio ha sido mucho más escaso (Maala et al., 2007; Mahdy et al., 2018; Savoldi et al., 2018).

A la vista de todo ello, el objetivo planteado por los autores fue comparar la morfometría del hueso palatino en la oveja (Ovis aries) y en la cabra (Capra hircus) domésticas, centrándonos en la lámina horizontal, que es la que forma parte del paladar duro. En nuestro estudio intentamos aportar datos sobre esta arquitectura ósea del cráneo en los pequeños rumiantes, y así contribuir al mejor conocimiento de la morfología ósea comparada en las especies domésticas. Hasta dónde llega el conocimiento de los autores, no se han realizado hasta el momento análisis morfométricos comparativos del hueso palatino en ovejas y cabras, aunque sí es más abundante la bibliografía para otras piezas óseas (Salvagno \& Albarella, 2017).

\section{MATERIAL Y MÉTODO}

Muestra. La muestra estaba compuesta por 53 cráneos limpios de ovejas $(n=36)$ y cabras $(n=17)$ adultos (al menos M2 erupcionada). Ninguno de los cráneos muestreados presentaba signos de deformación o trauma craneofacial. Como los huesos están separados por cartílago, pero osifican con la edad, los individuos seniles tampoco no fueron muestreados debido a la dificultad para delimitar claramente las suturas. El sexo no se consideró en este estudio, ya que el etiquetado de los especímenes no incluía esta información, aunque ningún espécimen procedía de animales castrados. Todos los especímenes están actualmente disponibles y accesibles en la osteoteca del Departament de Ciència Animal de la Universitat de Lleida, en Cataluña (España). Aunque el material asociado a este estudio es de tipo biológico, no se requirieron permisos para el estudio descrito, que además cumplía con las regulaciones sanitarias. Sin tener una dimensión etnológica, cabe decir que todos los cráneos ovinos pertenecen a la raza Navarra y los caprinos, a la Blanca de Rasquera $(n=4)$, Catalana $(n=2)$, Murciana $(n=4)$ y desconocida $(n=7)$.
Obtención de las imágenes. Cada cráneo fue fotografiado en alta resolución en vista ventral estandarizada con una cámara digital. Las imágenes fueron obtenidas con una cámara digital Nikon ${ }^{\circledR}$ D70 (resolución de imagen de 2.240 x 1.488 píxeles) equipada con una lente telefoto Nikon AF Nikkor® de 28$200 \mathrm{~mm}$. Se utilizó un patrón calibrado en cada imagen. Las imágenes se almacenaron posteriormente en formato JPG.

Selección de hitos. Para capturar la forma del hueso palatino, utilizamos una combinación de hitos y de semi-hitos anatómicos -puntos espaciados por distancia (Gunz \& Mitteroecker, 2013), en el contorno. Se eligieron un total de 32 puntos (4 hitos y 28semi-hitos) para analizar la lámina horizontal del hueso palatino (Fig. 1). Los hitos se situaron en los puntos más rostral (palatino orale, el de contacto con

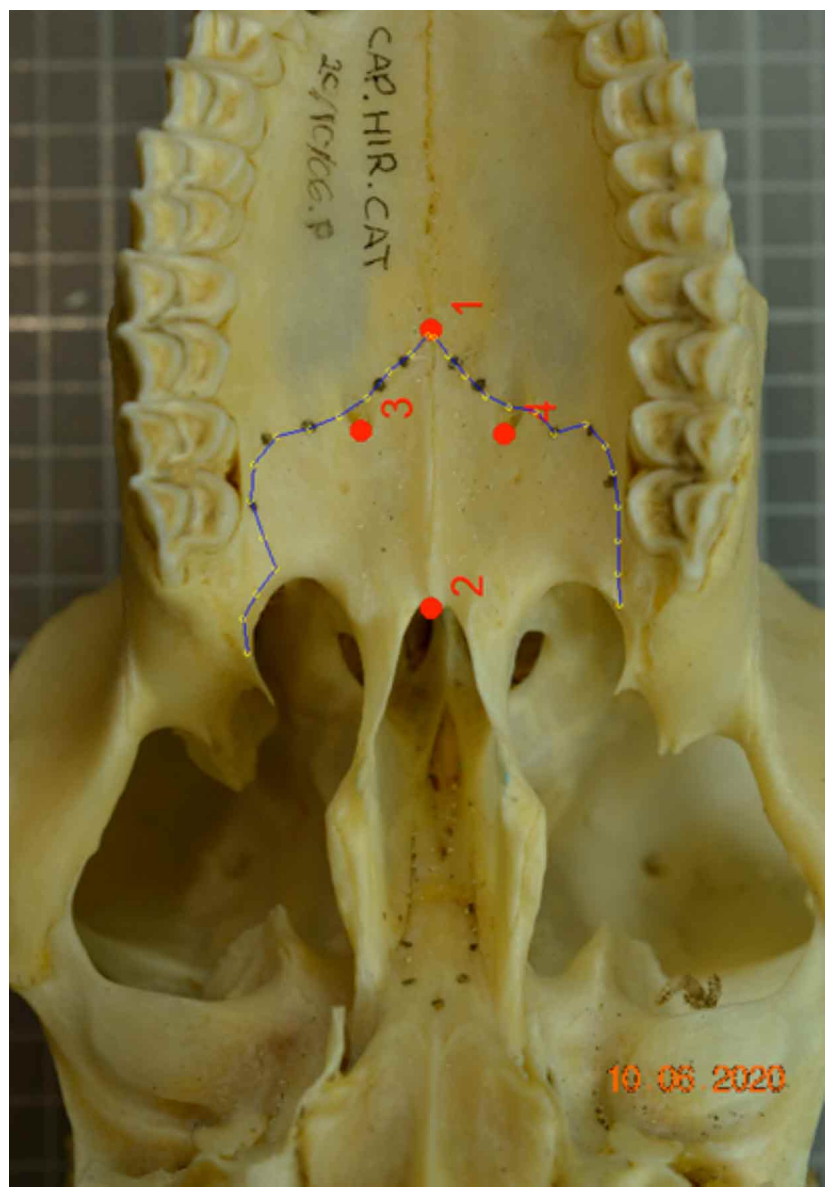

Fig. 1. Conjunto de puntos utilizados (4 hitos y 28 semi-hitos) para definir la lámina horizontal del hueso palatino mayor en Ovis y Capra. Los hitos se situaron en los puntos más rostral (palatinoorale, 1) y caudal (staphylion, 2) de la sutura palatina media, y en los puntos más caudales de los dos forámenes palatinos mayores ( 3 y 4). Los semi-hitos se situaron a lo largo de las suturas adyacentes rostral y laterales [sutura palatomaxillarisy sutura palatina transversa]. No se tuvieron en cuenta los forámenes palatinos menores. La imagen corresponde a una visión ventral de cráneo de cabra; la parte superior de la imagen es la rostral. 
el proceso palatino del maxilar) y caudal (staphylion, en el margen libre) de la sutura palatina mediana, y en los puntos más caudales de los dos forámenes palatinos mayores. Los semi-hitos se situaron a lo largo de las suturas adyacentes rostral y laterales [Sutura palatomaxillaris y Sutura palatina transversa], que previo a la fotografía se delimitaban en el cráneo. No se tuvieron en cuenta coordenadas sobre los forámenes palatinos menores, por la inconsistente presencia individual de ellos. Las coordenadas cartesianas $\mathrm{x}$ e y de todos los puntos se digitalizaron utilizando los programas TpsDig 2.04 v. 1.40 (Rohlf, 2015), y el programa Tps Util v. 1.70 (Rohlf) para el ajuste de curvas a partir de los semi-hitos. Puesto que el error puede explicar una fracción significativa de la varianza entre los lados y alterar los resultados, las digitalizaciones se realizaron dos veces para evaluar el error.

La primera fase del tratamiento de los datos incluyó la transformación de las coordenadas cartesianas mediante una superposición Procrustes generalizada. Esto permitió eliminar toda la variabilidad derivada de la escala, rotación y traslación. Puesto que este hueso es irregular y frecuentemente descrito como asimétrico, a partir de esta superposición se extrajo la matriz del componente simétrico de la variación, que representa la variación en forma entre los individuos y puede considerarse como el promedio de los lados derecho e izquierdo (Klingenberg et al., 2002).

Se realizó un primer análisis de regresión multivariante de las coordenadas de Procrustes a fin de detectar la posible presencia de alometría, entendida como el cambio de la forma en función del tamaño, expresado este como "tamaño de centroide", logarítmicamente transformado. Los análisis posteriores se realizaron con los residuales de la regresión. Seguidamente se procedió a comparar los tamaños mediante un testU de Mann-Whitney. Para la comparación de las formas se recurrió a un test NPMANOVA ("Non Parametric Multivarinate Analysis Of Variance") de dos vías, usando las distancias euclidianas y 9999 permutaciones. Los dos factores considerados fueron la réplica y el género. Un Análisis Discriminante (AD) nos permitió establecer los porcentajes de individuos incorrectamente asignados a un grupo o a otro. Y, finalmente, a fin de visualizar las diferencias entre ambos grupos, se procedió a obtener una rejilla de deformación ("thinplate") para cada género. Ello nos permitió identificar e interpretar visualmente el significado del cambio de forma en cada uno.

Los análisis estadísticos se realizaron con los programas MorphoJ v. 1.06c (Klingenberg, 2011) y PAST v. 2.17c (Hammer et al., 2001). Se estableció el nivel de confianza en el $95 \%$.

\section{RESULTADOS}

Error de medición. El test NPMANOVA no reflejó diferencias estadísticamente significativas entre ambas réplicas $(\mathrm{p}=0,854)$ (Tabla I), siendo el error de medición del 4,1\%. Estos datos muestran una importante precisión en las medidas, con diferencias escasas entre ambas especies, y subrayan la reproductibilidad del método.

Alometría. La alometría apareció estadísticamente significativa $(\mathrm{p}<0,02)$ tanto para Ovis como para Capra, con un $3,99 \%$ y un $8,11 \%$, respectivamente, del cambio de la forma debido al cambio del tamaño.

Diferencias entre ambos géneros. El test de Mann-Whitney reflejódiferencias estadísticamente significativas entre ambos grupos para el tamaño ( $U=670, p=0,000179)$, siendo el tamaño mayor en Capra. El test NPMANOVA reflejó igualmente diferencias estadísticamente significativas para la forma pura ( $\mathrm{p}=0,0001)$.Del $\mathrm{AD}$ se desprende que un 13,3\% de huesos de Capra fueron incorrectamente asignados a Ovis, y un $1,4 \%$ de Ovis, incorrectamente asignados a Capra. Las láminas de expansión/contracción aparecen en la Figura 2.

\section{DISCUSIÓN}

De nuestros resultados se desprende que en Capra se denota una clara expansión lateral y rostral del hueso palatino, mientras que en Ovis se presenta una tendencia a la contracción lateral y rostral. Dicho de un modo más colo-

Tabla I. Resultados del NPMANOVA de dos vías para la forma del hueso palatino de 53 cráneos de ovejas $(n=36)$ y cabras $(n=17)$ adultos.

\begin{tabular}{lccccc}
\hline Factor & $\begin{array}{c}\text { Sumatorio } \\
\text { de cuadrados }\end{array}$ & $\begin{array}{c}\text { Grados de } \\
\text { libertad }\end{array}$ & $\begin{array}{c}\text { Promedio } \\
\text { cuadrado }\end{array}$ & $\mathrm{F}$ & $p$ \\
\hline Género & 0,10245 & 1 & 0,102450 & 7,777 & 0,0001 \\
Réplica & 0,00573 & 1 & 0,005732 & 0,435 & 0,8543 \\
Interacción & $-0,16281$ & 1 & $-0,162810$ & $-12,361$ & 0,4074 \\
Residual & 1,34350 & 102 & 0,013172 & & \\
Total & 1,28890 & 105 & & & \\
\hline
\end{tabular}




\section{1/ Capra}

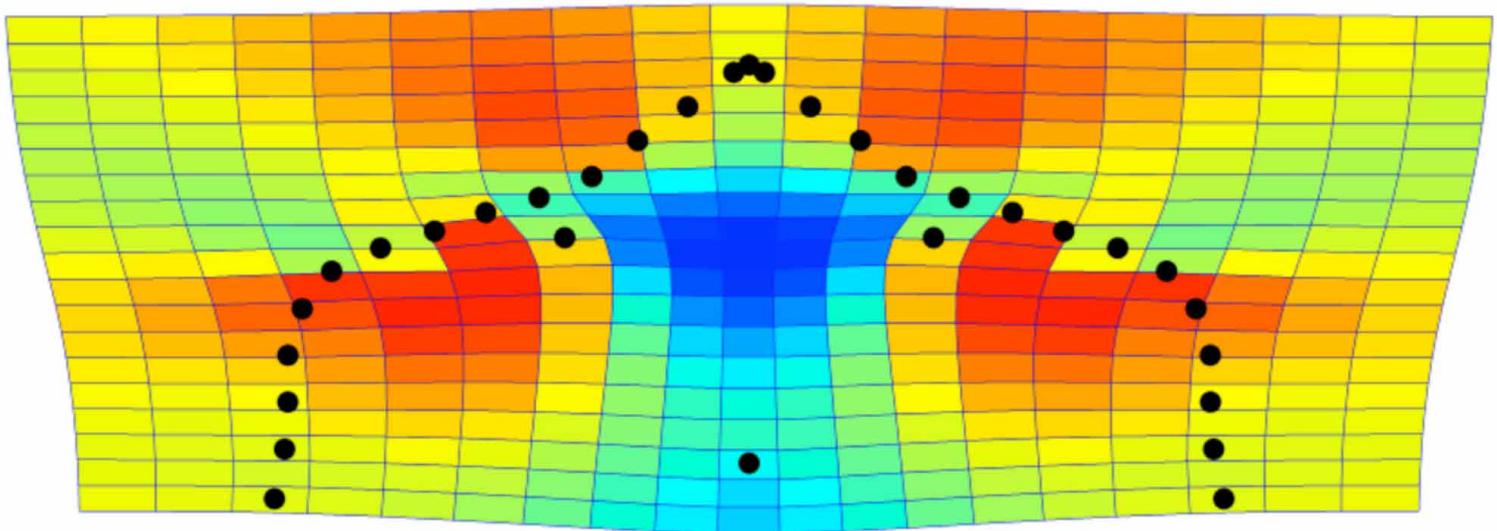

\section{2/ Ovis}

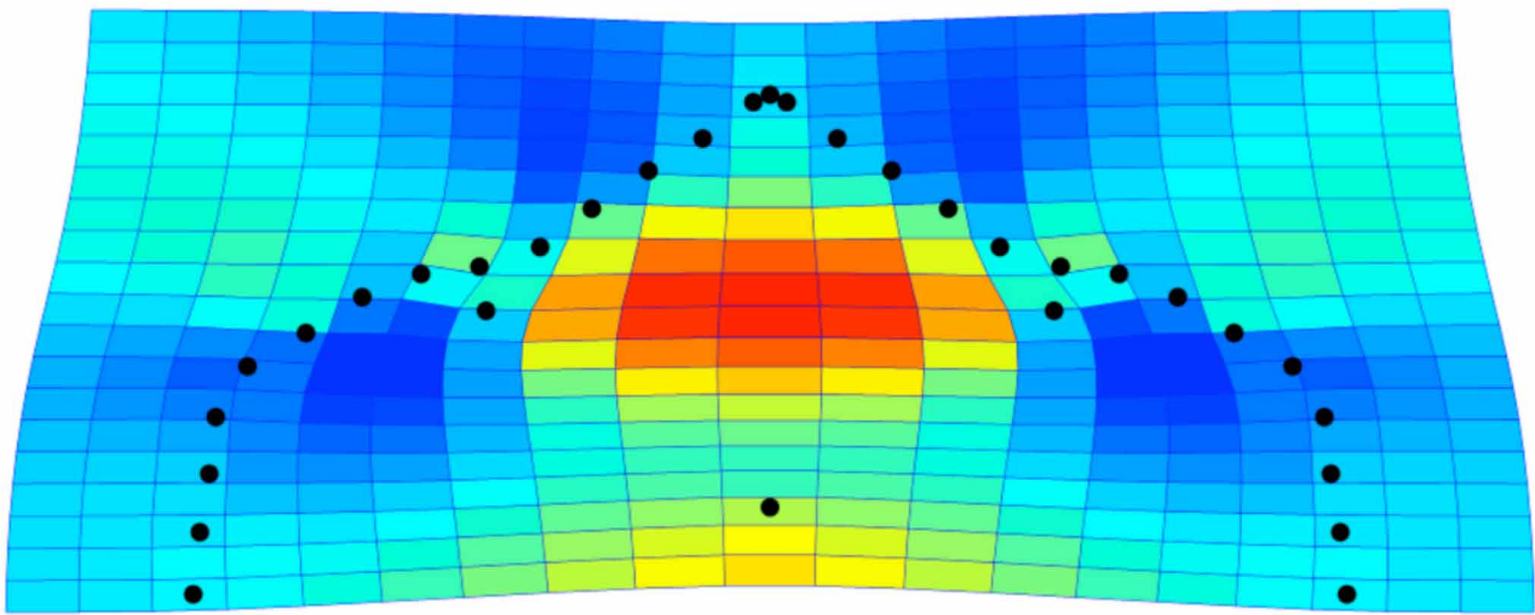

Fig. 2. Las láminas de expansión/contracción. Se emplean colores para representar los factores de expansión, indicando el grado de expansión o contracción direccional local. Las zonas rojas representan áreas de expansión, frente a las azules.

quial, el paladar de las cabras tendería a ser más largo y ancho que el de la oveja. La discriminación para diferenciar ambos géneros es menor en Capra.

La lengua participa en la masticación poniendo el alimento detrás de los dientes reagrupando al bolo alimenticio e impactándolo contra el paladar (Herring, 1993) (Halata et al., 1999). El paladar juega un rol pasivo en la masticación, pues da apoyo a la lengua para colocar el alimento bajo las arcadas yugales (Fuentes et al., 2018). Así, la lengua frota muy fuertemente el alimento con la parte rugosa palatina (Halata et al.) y, siendo la cabra una especie que suele ingerir alimentos groseros (Mahdy et al.), es coherente pensar que el paladar duro presente una mayor anchura y longitud, puesto que se traduciría en una mayor superficie de frotación. El tamaño de centroide (que geométricamente en este caso podría equipararse a la superficie) también en la cabra es comparativamente mayor que en la oveja. Así, esta mayor área de la lámina horizontal en Capra se explicaría por una mayor capacidad de frotación del alimento en relación a Ovis. A su vez, esta mayor capacidad de frotación responde a la necesidad debido al tipo de alimento que ingieren las cabras, mucho más áspero y leñoso que el que suele ingerir una oveja.

AGRADECIMIENTOS. Los autores agradecen los comentarios realizados por los revisores anónimos. 
PARÉS-CASANOVA, P. M.; DOMÈNECH, X.; ROJO, C. \& SAN ROMÁN, F. Morphometric palatine differences between sheep (Ovis aries) and goat (Capra hircus). Int. J. Morphol., 39(3):797-801, 2021.

SUMMARY: Palatine is a cranial bone very less studied in comparative veterinary anatomy. In our study, we performed the comparison of this bone between sheep (Ovis aries L., 1758) and goat (Capra hircus L., 1758), from a sample of 36 and 17 skulls respectively, using methods of geometric morphology. A total of 32 points (4 landmarks and 28 semi-landmarks) were chosen to analyse the horizontal lamina of the palatine bone. Statistically significant differences were reflected for both size and shape. Capra denotes a clear lateral expansion of the bone and rostral and a central contraction, with a relative approach of palatine foramina, while in Ovis foramina are more laterally distant. In our view, this greater width of the horizontal lamina in Capra would be explained by a greater capacity to rub the food compared to Ovis.

KEY WORDS: Blanca de Rasquera breed; Splanchnocranium; Hard palate; Navarra breed; Viscerocranium.

\section{REFERENCIAS BIBLIOGRÁFICAS}

Burcu, O.; Murat, K. \& Kerem, A. Anatomic and craniometric factors in differentiating roe deer (Capreolus capreolus) from sheep (Ovis aries) and goat (Capra hircus) skulls. Arch. Biol. Sci., 65(1):13341, 2013.

Choudhary, O. P.; Priyanka; Kalita, P. C.; Arya, R. S.; Kalita, A.; Doley, P. J. \& Keneisenuo. A morphometrical study on the skull of goat (Capra hircus) in Mizoram. Int. J. Morphol., 38(5):1473-8, 2020.

Dalga, S. Topographic and morphometric study of the mental foramina of Abaza goats with its clinical implication for regional anesthesia. Folia Morphol. (Warsz), 79(3):576-9, 2020.

Evans, K. E. \& McGreevy, P. D. Conformation of the equine skull: a morphometric study. Anat. Histol. Embryol., 35(4):221-7, 2006.

Fuentes, R.; Arias, A.; Lezcano, M. F.; Saravia, D.; Kuramochi, G.; Navarro, P. \& Dias, F. J. A new tridimensional insight into geometric and kinematic characteristics of masticatory cycles in participants with normal occlusion. Biomed Res. Int.. 2018:2527463, 2018.

Gedrange, T.; Köbel, C. \& Harzer, W. Hard palate deformation in an animal model following quasi-static loading to stimulate that of orthodontic anchorage implants. Eur. J. Orthod., 23(4):349-54, 2001.

Geiger, M. \& Haussman, S. Cranial suture closure in domestic dog breeds and its relationships to skull morphology. Anat. Rec. (Hoboken), 299(4):412-20, 2016.

Gioso, M. A. \& Carvalho, V. G. G. Oral anatomy of the dog and cat in veterinary dentistry practice. Vet. Clin. North Am. Small Anim. Pract., 35(4):763-80, 2005.

Goodarzi, N. \& Hoseini, T. S. Morphologic and osteometric analysis of the skull of Markhoz goat (Iranian Angora). Vet. Med. Int., 2014:972682, 2014.

Gunz, P. \& Mitteroecker, P. Semilandmarks: A Method for Quantifying Curves and Surfaces. Hystrix Ital. J. Mamm., 24(1):103-9, 2013.

Halata, Z.; Cooper, B. Y.; Baumann, K. I.; Schwegmann, C. \& Friedman, R. M. Sensory nerve endings in the hard palate and papilla incisiva of the goat. Exp. Brain Res., 129(2):218-28, 1999.

Hammer, Ø.; Harper, D. A. T. \& Ryan, P. D. PAST v. 2.17c. Palaeontol. Electron., 4(1):1-229, 2001.
Herring, S. W. Functional morphology of mammalian mastication. Am. Zool., 33(3):289-99, 1993.

Is s, eri, H.; Tekkaya, A. E.; Oztan, O. \& Bilgiç, S. Biomechanical effects of rapid maxillary expansion on the craniofacial skeleton, studied by the finite element method. Eur. J. Orthod., 20(4):347-56, 1998.

Klingenberg, C. P. MorphoJ: an integrated software package for geometric morphometrics. Mol. Ecol. Resour, 11(2):353-7, 2011.

Klingenberg, C. P.; Barluenga, M. \& Meyer, A. Shape analysis of symmetric structures: quantifying variation among individuals and asymmetry." Evolution, 56(10):1909-20, 2002.

König, H. E. \& Liebich, H. G. Anatomía de los Animales Domésticos. Texto y Atlas en Color. Tomo 1. Aparato Locomotor. Madrid, Médica Panamericana, 2005.

Laganà, G.; Di Fazio, V.; Paoloni, V.; Franchi, L.; Cozza, P. \& Lione, R. Geometric morphometric analysis of the palatal morphology in growing subjects with skeletal open bite. Eur. J. Orthod., 41(3):258-63, 2019.

Lee, S. P.; Paik, K. S. \& Kim, M. K. Anatomical study of the pyramidal process of the palatine bone in relation to implant placement in the posterior maxilla. J. Oral Rehabil., 28(2):125-32, 2001.

Maala, C. P.; Ducusin, R. J. T. \& Rizori, J. A. The gross anatomy of the hard palate and palatine printing in cattle. Philipp. J. Vet. Med., 44(1):17, 2007.

Mahdy, M. A. A.; Abdalla, K. E. H. \& Mohamed, S. A. Morphological study of the hard palate in the Egyptian goats (Capra hircus): A scanning electron microscopic study. Anat. Histol. Embryol., 47(5):391-7, 2018.

Merkies, K.; Paraschou, G. \& McGreevy, P. D. Morphometric characteristics of the skull in horses and donkeys-A pilot study. Animals (Basel), 10(6):1002, 2020.

Parés-Casanova, P. M. Geometric morphometrics to the study of skull sexual dimorphism in a local domestic goat breed. J. Fish. Livest. Prod., 3(3):14, 2015.

Rodrigues, R. T. S.; Matos, W. C. G.; Walker, F. M.; Costa, F. S.; Wanderley, C. W. S.; Pereira Neto, J. \& Faria, M. D. Dimensions of the cranium and of the cranial cavity and intracranial volume in goats (Capra hircus LINNAEUS, 1758). J. Morphol. Sci., 27(1):6-10, 2010.

Rohlf, F. J. The Tps Series of Software. Hystrix Ital. J. Mamm., 26(1):1-4, 2015.

Salvagno, L. \& Albarella, U. A morphometric system to distinguish sheep and goat postcranial bones. PLoS One, 12(6):e0178543, 2017.

Savoldi, F.; Xu, B.; Tsoi, J. K. H.; Paganelli, C. \& Matinlinna, J. P. Anatomical and mechanical properties of swine midpalatal suture in the premaxillary, maxillary, and palatine region. Sci. Rep., 8(1):7073, 2018.

Schoenebeck, J. J. \& Ostrander, E. A. The genetics of canine skull shape variation. Genetics, 193(2):317-25, 2013.

Sisson, S. \& Grossman, G. D. Anatomía de los Animales Domésticos. Tomo I. Barcelona, Salvat, 1985.

Dirección para correspondencia:

Pere M. Parés-Casanova

Departamento de Ciencia Animal, ETSEA

Universidad de Lleida

Av. Rovira Roure 191, 25198 Lleida

Cataluña

ESPAÑA

Email: peremiquelp@ca.udl.cat

Recibido : 09-01-2021

Aceptado: 22-03-2021 